\title{
Chest Circumference
}

National Cancer Institute

\section{Source}

National Cancer Institute. Chest Circumference. NCI Thesaurus. Code C156606.

A circumferential measurement of the chest. 